\title{
Enhancer transcription: what, where, when, and why?
}

\author{
Nathaniel D. Tippens, ${ }^{1,2}$ Anniina Vihervaara, ${ }^{1}$ and John T. Lis ${ }^{1,2}$ \\ ${ }^{1}$ Department of Molecular Biology and Genetics, Cornell University, Ithaca, New York 14853, USA; ${ }^{2}$ Tri-Institutional Training \\ Program in Computational Biology and Medicine, Cornell University, Ithaca, New York 14853, USA
}

Following the discovery of widespread enhancer transcription, enhancers and promoters have been found to be far more similar than previously thought. In this issue of Genes \& Development, two studies (Henriques and colleagues [pp. 26-41] and Mikhaylichenko and colleagues [pp. 42-57]) shine new light on the transcriptional nature of promoters and enhancers in Drosophila. Together, these studies support recent work in mammalian cells that indicates that most active enhancers drive local transcription using factors and mechanisms similar to those of promoters. Intriguingly, enhancer transcription is shown to be coordinated by SPT5- and P-TEFb-mediated pauserelease, but the pause half-life is shorter, and termination is more rapid at enhancers than at promoters. Moreover, bidirectional transcription from promoters is associated with enhancer activity, lending further credence to models in which regulatory elements exist along a spectrum of promoter-ness and enhancer-ness. We propose a general unified model to explain possible functions of transcription at enhancers.

Enhancers are regulatory elements that activate promoter transcription over large distances and independently of orientation (Serfling et al. 1985). While both promoters and enhancers are known to bind transcription factors (TFs), only promoters were thought to initiate transcription by RNA polymerase II (Pol II). With the advent of high-throughput sequencing, molecular features at enhancers and promoters have been revealed in unprecedented detail: Enhancers produce RNAs (eRNAs) in vivo (Kim et al. 2010) with an initiation and chromatin architecture remarkably similar to that of promoters (Core et al. 2014; Scruggs et al. 2015). This has greatly renewed interest in the overlooked finding that mammalian enhancer activity can co-occur with promoter activity (Serfling et al. 1985; Arnold et al. 2013; Dao et al. 2017). In this issue of Genes \& Developement, two reports further clarify the role of enhancer transcription. Henriques

[Keywords: promoters; eRNA; embryonic development; enhancers; P-

$\mathrm{TEFb}$; superenhancers; transcription; termination]

Corresponding author: johnlis@cornell.edu

Article is online at http://www.genesdev.org/cgi/doi/10.1101/gad.311605. 118 . et al. (2018) perform detailed genome-wide analyses of pausing and termination at unannotated transcription start sites (TSSs) and show a striking overlap with enhancers identified previously by the episomal STARR-seq (self-transcribing active regulatory region [STARR] with sequencing) assay (Arnold et al. 2013). Mikhaylichenko et al. (2018) compare transcriptional strength and directionality with enhancer and promoter activities in vivo. Both studies rely on short capped RNA sequencing techniques-Start-seq and PRO-cap (a precision nuclear runon sequencing variant) - that identify TSSs across the genome with high sensitivity.

Henriques et al. (2018) offer a detailed characterization of enhancer transcription in Drosophila S2 cells by comparing production of short capped RNAs to enhancer activity. The investigators found that $49 \%$ of Start-seqidentified previously unannotated TSSs overlap with STARR-seq-called enhancers. Furthermore, $94.2 \%$ of enhancers found within accessible chromatin in vivo show at least five RNA reads, consistent with most enhancers driving some level of transcription. The level of short capped RNAs showed moderate correlation with episomal enhancer activity $(\rho=0.24)$, suggesting some quantitative connection between enhancer transcription and activity. Interestingly, the investigators report that most enhancers are divergently or convergently transcribed or both.

Similar results were obtained by Mikhaylichenko et al. (2018), who investigated where and when enhancer transcription and enhancer activity occur in Drosophila embryos. By generating whole-embryo PRO-cap and CAGE (cap analysis of gene expression) data at matched time points, the investigators compare transcription with transgenic reporter activity at thousands of previously characterized developmental enhancers. The results validate the concept that enhancer transcription generally coincides with its functional activity and that active enhancers can possess a range of transcription levels and directionalities. The inability to detect eRNA production from every functional enhancer leaves open the possibility

(C) 2018 Tippens et al. This article is distributed exclusively by Cold Spring Harbor Laboratory Press for the first six months after the full-issue publication date (see http://genesdev.cshlp.org/site/misc/terms.xhtml). After six months, it is available under a Creative Commons License (Attribution-NonCommercial 4.0 International), as described at http:// creativecommons.org/licenses/by-nc/4.0/. 
for untranscribed but active enhancers in vivo or may simply reflect the reduced sensitivity of whole-embryo assays. The concept of diverse enhancer mechanisms is supported by the variety of changes in transcription and chromatin detected upon induced TF binding (Vihervaara et al. 2017).

To quantify transcriptional strength and directionality from regulatory elements, Mikhaylichenko et al. (2018) developed an elegant transgenic assay with dual vectors that simultaneously assess the element's ability to function as an enhancer and a promoter in vivo. In a limited set of representative elements, most enhancers with bidirectional transcription functioned as weak promoters in both directions. This promoter activity occurred predominantly in the same tissue or subset of cells as the elements' endogenous enhancer activity, indicating that enhancers and promoters depend on the same regulatory components. Furthermore, promoters with bidirectional transcription harbored some enhancer activity, appearing to function as an enhancer and promoter for the same gene, similar to recent results in human cells (Dao et al. 2017). In contrast, the majority of promoters with unidirectional transcription did not show enhancer activity, and the endogenous direction of transcription correlated with the orientation in which the element functioned as a promoter. Together, these results are consistent with upstream promoter sequences and their upstream antisense TSSs behaving similarly to distal enhancers (Serfling et al. 1985; Arnold et al. 2013; Scruggs et al. 2015; Dao et al. 2017).

The unified chromatin architecture and transcription at promoters and enhancers suggests shared mechanisms of regulation (Core et al. 2014). For example, one of the major rate-limiting steps at most promoters is pause-release, regulated by SPT5 and P-TEFb. Henriques et al. (2018) demonstrate that these same factors regulate pausing at enhancers. Importantly, the study provides elegant timecourse data that estimates pause half-lives genome-wide and demonstrates less stable pausing at enhancers than promoters. Furthermore, sequencing oligo-adenylated intermediates from exosome-deficient cells uncovered premature termination at enhancers, suggesting that enhancer activity may rely on local recycling of terminated Pol II. Finally, the investigators report that in mouse ESCs, "superenhancers" and many promoters contain large clusters of TSSs, indicating similar regulatory mechanisms at these loci. Intriguingly, such sites have extremely rapid pause-release, perhaps driven by high local concentrations of $\mathrm{P}-\mathrm{TEFb}$, and thus appear resistant to loss of pausing factors. Altogether, Henriques et al. (2018) confirm and extend our mechanistic understanding of initiation, pausing, and termination and clarify patterns of histone modification and lineage-defining TFs at enhancers.

Profound challenges in the field remain to be resolved to further clarify enhancer mechanisms. A major challenge is to rigorously assign functional enhancer-promoter connections and quantify enhancer strength with regard to each target gene in its endogenous context. Too often, we have to rely on the "closest gene estimate," which is inadequate in vivo (Fulco et al. 2016). Another challenge is identifying enhancers genome-wide. Henriques et al. (2018) show convincingly that the H3K4me1/H3K4me3 ratio fails to identify highly transcribed enhancers, consistent with reports in mammalian cells (Core et al. 2014; Dao et al. 2017). These results indicate that enhancers are difficult to distinguish from promoters by histone modification patterns alone and highlight the utility of using unstable bidirectional transcription for enhancer identification. The features and mechanisms that specify rapid Pol II termination and eRNA instability at these sites remain to be fully identified.

Enhancers and promoters share many features, including similar sequence motifs, transcription machinery, chromatin environment, and changes in activity upon binding of activators or repressors (Core et al. 2014; Scruggs et al. 2015; Fulco et al. 2016; Vihervaara et al. 2017). However, the functional role of transcription from enhancers remains elusive. It is tempting to speculate that transcription itself helps mediate enhancer-promoter colocalization, perhaps through Pol II's affinity for common coactivators such as Mediator, CBP, Integrator, remodeling complexes, and histone modifiers. Alternatively, transcription may simply maintain open and active chromatin architecture (for example, Scruggs et al. 2015), thus allowing enhancer-promoter interactions through factor binding. Either model of transcriptiondriven enhancer and promoter connectivity helps to explain their extreme similarities in initiation and pausing behaviors.

\section{Acknowledgments}

We apologize to authors whose work could not be cited in this brief communication. This work was supported by the Center for Vertebrate Genomics through National Institutes of Health (NIH) training grant T32HD057854 to N.D.T., the Sigrid Jusélius Foundation to A.V., and NIH UM1HG009393 to J.T.L. The content is solely the responsibility of the authors and does not necessarily represent the official views of the National Institute of Health.

\section{References}

Arnold CD, Gerlach D, Stelzer C, Boryń ŁM, Rath M, Stark A. 2013. Genome-wide quantitative enhancer activity maps identified by STARR-seq. Science 339: 1074-1077.

Core LJ, Martins AL, Danko CG, Waters CT, Siepel A, Lis JT. 2014. Analysis of nascent RNA identifies a unified architecture of initiation regions at mammalian promoters and enhancers. Nat Genet 46: 1311-1320.

Dao LT, Galindo-Albarrán AO, Castro-Mondragon JA, AndrieuSoler C, Medina-Rivera A, Souaid C, Charbonnier G, Griffon A, Vanhille L, Stephen T, et al. 2017. Genome-wide characterization of mammalian promoters with distal enhancer functions. Nat Genet 49: 1073-1081.

Fulco CP, Munschauer M, Anyoha R, Munson G, Grossman SR, Perez EM, Kane M, Cleary B, Lander ES, Engreitz JM. 2016. Systematic mapping of functional enhancer-promoter connections with CRISPR interference. Science 354: 769773. 
Henriques T, Scruggs BS, Inouye MO, Muse GW, Williams L, Burkholder AB, Lavender CA, Fargo DC, Adelman K. 2018. Widespread transcriptional pausing and elongation control at enhancers. Genes Dev (this issue). doi: 10.1101/ gad.309351.117.

Kim TK, Hemberg M, Gray JM, Costa AM, Bear DM, Wu J, Harmin DA, Laptewicz M, Barbara-Haley K, Kuersten S, et al. 2010. Widespread transcription at neuronal activity-regulated enhancers. Nature 465: 182-187.

Mikhaylichenko O, Bondarenko V, Harnett D, Schor IE, Males M, Viales RR, Furlong EEM. 2018. The degree of enhancer or promoter activity is reflected by the levels and directionality of
eRNA transcription. Genes Dev (this issue). doi: 10.1101/ gad.308619.117.

Serfling E, Jasin M, Schaffner W. 1985. Enhancers and eukaryotic gene transcription. Trends Genet 1: 224-230.

Scruggs BS, Gilchrist DA, Nechaev S, Muse GW, Burkholder A, Fargo DC, Adelman K. 2015. Bidirectional transcription arises from two distinct hubs of transcription factor binding and active chromatin. Mol Cell 58: 1101-1112.

Vihervaara A, Mahat DB, Guertin MJ, Chu T, Danko CG, Lis JT, Sistonen L. 2017. Transcriptional response to stress is prewired by promoter and enhancer architecture. Nat Commun 8: 255. 


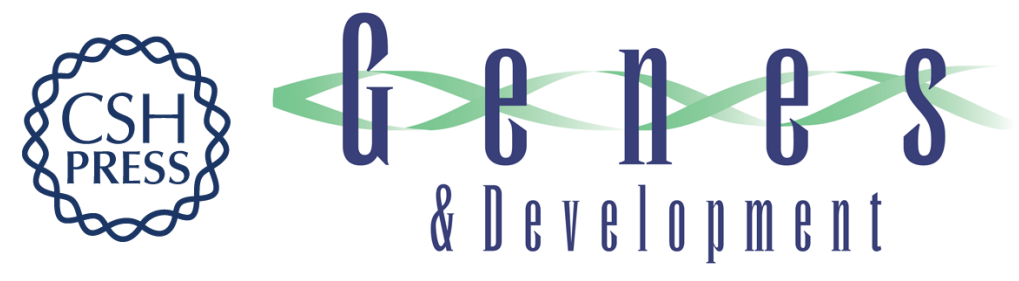

\title{
Enhancer transcription: what, where, when, and why?
}

\author{
Nathaniel D. Tippens, Anniina Vihervaara and John T. Lis
}

Genes Dev. 2018, 32:

Access the most recent version at doi:10.1101/gad.311605.118

\section{Related Content Widespread transcriptional pausing and elongation control at enhancers \\ Telmo Henriques, Benjamin S. Scruggs, Michiko O. Inouye, et al. Genes Dev. January, 2018 32: 26-41 The degree of enhancer or promoter activity is reflected by the levels and directionality of eRNA transcription Olga Mikhaylichenko, Vladyslav Bondarenko, Dermot Harnett, et al. Genes Dev. January, 2018 32: 42-57}

References This article cites 10 articles, 4 of which can be accessed free at: http://genesdev.cshlp.org/content/32/1/1.full.html\#ref-list-1

Articles cited in: http://genesdev.cshlp.org/content/32/1/1.full.html\#related-urls

Creative This article is distributed exclusively by Cold Spring Harbor Laboratory Press for the first Commons License six months after the full-issue publication date (see http://genesdev.cshlp.org/site/misc/terms.xhtml). After six months, it is available under a Creative Commons License (Attribution-NonCommercial 4.0 International), as described at http://creativecommons.org/licenses/by-nc/4.0/.

\section{Email Alerting Service} Receive free email alerts when new articles cite this article - sign up in the box at the top right corner of the article or click here.

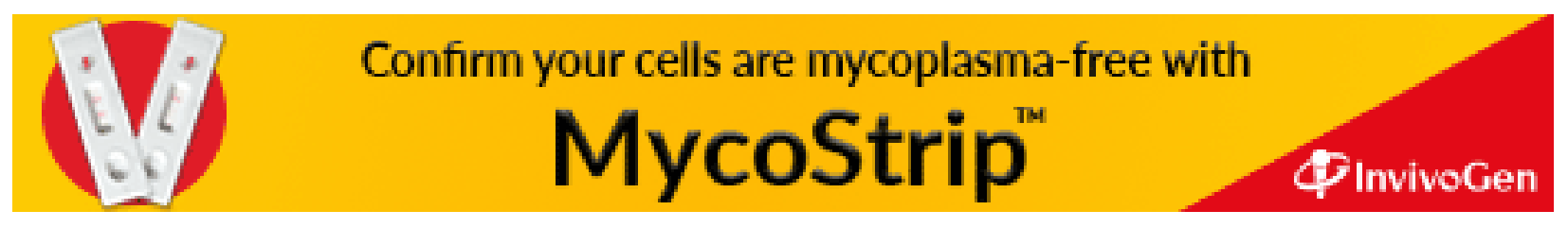

